\title{
Responsabilidades do enfermeiro no manuseio e cuidados com cateter em pacientes oncológicos: uma revisão integrativa
}

O objetivo deste trabalho é discorrer sobre a importância da manipulação e manuseio correto pelo enfermeiro em cateteres nos pacientes oncológicos. Trata-se de um estudo de revisão integrativa da literatura. Realizou-se a busca de artigos científicos nas bases de dados LILACS, BDENF e REUOL, publicados entre os anos de 2011 a 2021. Os resultados são apresentados por meio de fluxograma e tabela comparativa. Foram encontrados 235 artigos, analisaram-se 39 e, destes, 8 foram selecionados. Na síntese da discussão observou-se a importância da execução da Sistematização da Assistência de Enfermagem (SAE), prática de educação continuada pertinentes aos cateteres utilizados em oncologia, bem como a inserção de protocolo e diretrizes, atualização técnico-científica do enfermeiro e treinamento de equipe. A prática clínica em pacientes oncológicos portadores de dispositivos intravasculares se mostrou deficitária, na difusão de informações, o conhecimento do enfermeiro demonstrou-se restrito, e apresenta a importância de educação continuada, efetivação de protocolos a fim de garantir assistência de qualidade e evitar complicações.

Palavras-chave: Cateteres; Oncologia; Enfermagem; Segurança do Paciente; Infecções Relacionadas a Cateter.

\section{Nurses responsibilities in the handling and care of catheters in oncology patients: an integrative review}

The objective is to discuss the importance of correct manipulation and handling by nurses of catheters in oncology patients. This is an integrative literature review study. A search for scientific articles in the LILACS, BDENF and REUOL databases, published between 2011 and 2021 , was conducted. The results are presented by means of a flowchart and comparative table. 235 articles were found, 39 were analyzed and, of these, 8 were selected. In the synthesis of the discussion, it was observed the importance of the implementation of the Systematization of Nursing Care (SAE), the practice of continuing education relevant to the catheters used in oncology, as well as the insertion of protocols and guidelines, technical-scientific updating of nurses, and team training. The clinical practice in oncologic patients with intravascular devices showed to be deficient, in the diffusion of information, the nurse's knowledge proved to be restricted, and presents the importance of continued education, effectiveness of protocols in order to guarantee quality assistance and avoid complications.

Keywords: Catheters; Oncology; Nursing; Patient Safety; Catheter Related Infections.

Topic: Enfermagem Geral

Reviewed anonymously in the process of blind peer

Rafael Henrique Lopes (iD)

Universidade de Sorocaba, Brasil

http://lattes.cnpq.br/4395322971584109

http://orcid.org/0000-0003-0369-2463

rafael-06henrique@hotmail.com

Ebraim Kelvin Floriano

Universidade de Sorocaba, Brasil

http://lattes.cnpq.br/7739599698126726

http://orcid.org/0000-0002-1836-3315

braimkel@hotmal.com

Débora Cabral Nunes Polaz (ic)

Universidade de Sorocaba, Brasil

http://lattes.cnpq.br/9342411050092970

http://orcid.org/0000-0003-4800-672X

debora.polaz@gmail.com
Received: 10/06/2021

Approved: 08/07/2021
Leandro Aparecido Souza (ID)

Universidade de Sorocaba, Brasil

http://lattes.cnpq.br/6090315154831086

http://orcid.org/0000-0001-8828-9918

leandroapsouza14@gmail.com
Referencing this:

LOPES, R. H.; FLORIANO, E. K.; POLAZ, D. C. N.; SOUZA, L. A.. Responsabilidades do enfermeiro no manuseio e cuidados com cateter em pacientes oncológicos: uma revisão integrativa. Scire Salutis, v.11, n.3, p.14-19, 2021. DOI: http://doi.org/10.6008/CBPC2236-9600.2021.003.0003 


\section{INTRODUÇÃO}

A necessidade da melhor alternativa na escolha do tratamento quimioterápico antineoplásico, bem como as vias de administração, prevenções de agravos e cuidados aos dispositivos vasculares ao paciente oncológico são fundamentais. As drogas quimioterápicas quando infundidas, devido ao alto teor vesicante ao vaso sanguíneo, sua infusão pode ser observada por algumas possibilidades de lesões, fragilidades vasculares, flebite, eritema, dor e enrijecimento dos vasos. Em relevância a esses aspectos de danos ao paciente, a implantação do cateter venoso central de longa permanência semi-implantado ou totalmente implantado, geralmente são os mais utilizados em oncologia (BORTOLI et al., 2019)

O fornecimento de acesso seguro para a realização de procedimentos como a quimioterapia se faz por meio do tipo de dispositivo utilizado. Os dispositivos de acesso venoso central (DAVC) frequentemente são confeccionados em silicone ou poliuretano, pois contribuem para manutenção da integridade da rede venosa do paciente. A manutenção da permeabilidade desses cateteres é crucial para a qualidade de vida desses pacientes, e o enfermeiro é responsável por esta manutenção (FONSECA et al., 2019) Pacientes que utilizam DAVC por longos períodos tendem a ter complicações como obstrução parcial ou total do DAVC, situações essas que levam a necessidade de substituição desses dispositivos (SANTOS et al., 2020).

A indicação do cateter ocorre de acordo com cada caso clínico, levando em consideração o tipo de tratamento, tempo de permanência, estadiamento e singularidade do paciente. A utilização de protocolos, e seu cumprimento é fundamental para garantir a qualidade do cuidado prestado, promovendo uma prática assistencial segura e efetiva (BARBOSA et al., 2017).

A manipulação desses dispositivos é de extrema complexidade, visto que, o manejo incorreto pode ocasionar diversas complicações, portanto há necessidade de uma expertise para a manipulação adequada, pois demanda conhecimento técnico-científico, farmacológico, fisiopatológico do câncer e dos cateteres, sendo assim, atribui-se como atividade privativa do enfermeiro, de acordo com a lei federal $n^{\circ} 7.498 / 1986$ (ALCÂNTARA et al., 2019).

O presente estudo tem como objetivos identificar e definir os dispositivos vasculares comumente utilizados em oncologia. Demonstrar, através da literatura, as responsabilidades do enfermeiro na manipulação desses cateteres em pacientes oncológicos, identificando as lacunas e complicações decorrentes ao uso desses dispositivos em pacientes com câncer e o nível de experiência técnico-científica relacionado ao manuseio dos mesmos.

\section{METODOLOGIA}

Trata-se de uma revisão integrativa da literatura realizada entre os meses de março e maio de 2021, a partir de busca eletrônica por artigos científicos disponíveis nas bases de dados científicas: Literatura Latino-Americana e do Caribe em Ciências em Saúde (LILACS); Base de Dados de Enfermagem (BDENF), Scientific Electronic Library Online (SCIELO) e Revista de Enfermagem UFPE On Line (REUOL) publicados no período de 2011 a 2021.

Utilizaram-se como estratégia de busca os vocábulos constantes nos Descritores em Ciências da 
Saúde (DeCS): Cateteres, Oncologia, Enfermagem, Segurança do Paciente, Infecções Relacionadas a Cateter e Permeabilidade. A busca ocorreu por meio do cruzamento dos descritores, tendo como conexão o termo "AND". Para a consolidação dos estudos seguiu-se as seguintes etapas: escolha do tema, levantamento bibliográfico preliminar, formulação do problema, busca de fontes, leitura dos resumos e leitura na íntegra dos artigos selecionados para esta pesquisa, organização lógica do assunto, e a redação do texto. Neste contexto, questiona-se "Qual é a atuação do enfermeiro frente à manutenção e cuidado de possíveis complicações na utilização de cateteres em oncologia?"

Utilizou-se como critérios de inclusão estudos originais; de acesso livre; publicados em forma de artigo; texto completo; disponíveis no idioma português; com intervalo de publicação de 2011 a 2021; que abordassem a temática: cateteres em oncologia, independente de faixa etária, gênero, e tipo do cateter. Os critérios de exclusão adotados: estudos com indisponibilidade de acesso; que não contemplavam ao objetivo da pesquisa; dissertações ou teses; administração de medicamentos, perfil epidemiológico, trabalhos incompletos e os que foram publicados anteriormente a 2011.

Devido à escassez de estudos publicados, houve a necessidade da efetivação de novas buscas, a fim de fomentar as possibilidades de ampliação da pesquisa, feito por meio dos descritores: Segurança do Paciente, Infecção Relacionada a Cateter e Permeabilidade. O cruzamento dos descritores seguiu o mesmo critério atribuído a primeira etapa de pesquisa. A busca total resultou em 235 artigos levantados, dos quais, após análise, foram descartados 227, que não atendiam aos critérios de inclusão, sendo selecionados 8 artigos.

Após a seleção dos trabalhos procedeu-se as leituras exploratórias, seletiva, analítica e por fim a redação do trabalho. Foram analisadas seis categorias dos artigos: descritores, título, autores, ano de publicação, tipo de estudo e resultados de cada estudo.

\section{RESULTADOS E DISCUSSÃO}

O fluxograma referido abaixo se trata do modelo utilizado para realização das três pesquisas pertinentes ao estudo. Nesse sentido, a divisão dos descritores encontra-se em escala de cores para elucidação das etapas referente à construção do artigo, como síntese das principais informações obtidas.

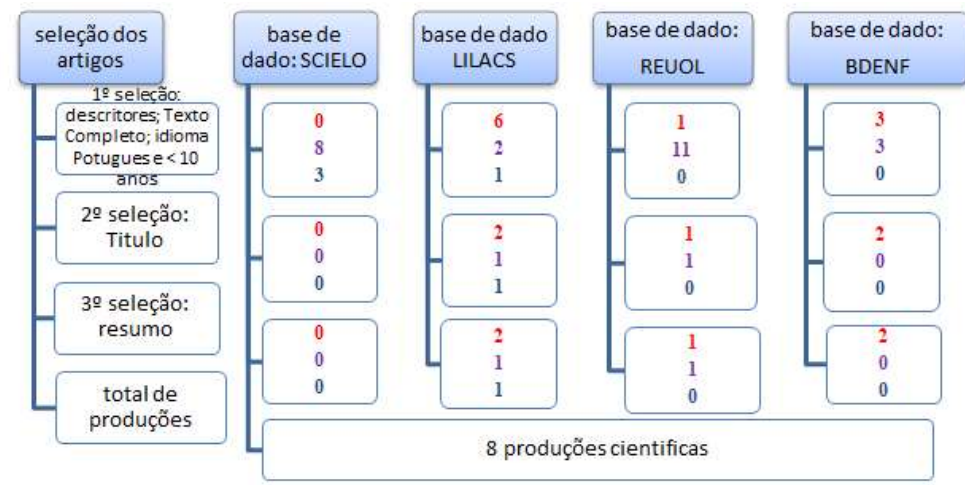

Fluxograma 1: Como se deu a busca em cada base de dados através dos descritores booleanos Cateteres e Oncologia e Enfermagem; Infecções Relacionadas a Cateter e Segurança do Paciente; Permeabilidade e Segurança do Paciente

A composição dos oito artigos selecionados, após a elaboração do fluxograma, são apresentados no 
Quadro 1.

Quadro 1: Caracterização da produção cientifica analisada segundo Descritores, Título do estudo, autores, anos de publicação, tipo de estudo e resultados, 2021

\begin{tabular}{|c|c|c|c|c|c|}
\hline DeCS & TÍTULO & AUTOR & NO & $\begin{array}{ll}\text { TIPO } & \text { DE } \\
\text { ESTUDO } & \end{array}$ & RESULTADOS \\
\hline \multirow{5}{*}{ 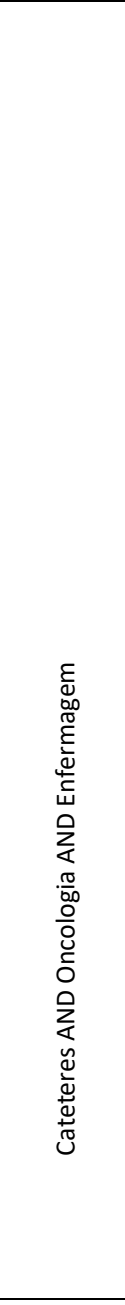 } & $\begin{array}{l}\text { Cateter Central de } \\
\text { Inserção Periférica: } \\
\text { Contribuições para } \\
\text { Enfermagem } \\
\text { Oncológica }\end{array}$ & $\begin{array}{l}\text { (ALCÂNTARA et } \\
\text { al., 2019) }\end{array}$ & 019 & $\begin{array}{l}\text { Estudo } \\
\text { Bibliográfico } \\
\text { tipo Revisão } \\
\text { Integrativa }\end{array}$ & $\begin{array}{l}\text { Com uma totalidade de } 455 \text { artigos pesquisados, sendo } \\
\text { analisados } 175 \text { onde foram selecionados } 15 \text { para a construção } \\
\text { do estudo, o mesmo foi sintetizado em duas categorias: } \\
\text {-A importância do conhecimento do profissional de Enfermagem } \\
\text { para o uso do PICC/CCIP } \\
\text {-A segurança do paciente por meio da prática segura } \\
\text { O enfermeiro tem autonomia para inserção; manipulação e } \\
\text { remoção do cateter de PICC/CCIP, pela resolução } 258 \text {, visto que } \\
\text { para esta realização o profissional deverá submeter- se à } \\
\text { qualificação ou capacitação especifica. }\end{array}$ \\
\hline & $\begin{array}{l}\text { Cateter Central de } \\
\text { Inserção Periférica } \\
\text { em Oncologia } \\
\text { Pediátrica: Um } \\
\text { Estudo } \\
\text { Retrospectivo }\end{array}$ & $\begin{array}{l}\text { (SANTANA et } \\
\text { al., 2018) }\end{array}$ & 018 & $\begin{array}{l}\text { Estudo } \\
\text { Retrospectivo }\end{array}$ & $\begin{array}{l}\text { Estudo realizado em hospital com especialidade pediátricas, } \\
\text { com abordagem de } 51 \text { prontuários de crianças e adolescente } \\
\text { que constavam a identificação e perfil desses pacientes em uso } \\
\text { de PICC. Remoção do cateter por término do tratamento }(45 \%) \text {, } \\
\text { infecções }(17,6 \%) \text {, tração acidental }(15,6 \%) \text {, obstrução }(11,7 \%) \text {, } \\
\text { óbito do paciente }(5,8 \%) \text { e ruptura de cateter }(3,9 \%) \text {. }\end{array}$ \\
\hline & $\begin{array}{l}\text { Infecção } \\
\text { Relacionada A } \\
\text { Cateter Venoso } \\
\text { Central: Indicador } \\
\text { de Qualidade Da } \\
\text { Assistência Em } \\
\text { Oncologia }\end{array}$ & $\begin{array}{l}\text { (MENEZES et } \\
\text { al., 2013) }\end{array}$ & 013 & $\begin{array}{l}\text { Estudo } \\
\text { exploratório }\end{array}$ & $\begin{array}{l}\text { Estudo feito através do INCA, com } 36 \text { pacientes que eram } \\
\text { portadores do Cateter Venoso Central de Longa Permanência. O } \\
\text { artigo traz em discussão e fundamenta os indicadores de } \\
\text { qualidade da assistência e processos correlacionados da } \\
\text { enfermagem em oncologia. Periodicidade da renovação de } \\
\text { curativos, manutenção da permeabilidade do cateter, } \\
\text { manutenção do sistema de infusão fechado e atenção aos sinais } \\
\text { flogísticos. }\end{array}$ \\
\hline & $\begin{array}{l}\text { Complicações } \\
\text { associadas ao uso } \\
\text { de Cateter } \\
\text { Totalmente } \\
\text { Implantável em } \\
\text { Crianças e } \\
\text { Adolescentes } \\
\end{array}$ & $\begin{array}{l}\text { (ORTOLANI et } \\
\text { al., 2013) }\end{array}$ & 013 & $\begin{array}{l}\text { Pesquisa } \\
\text { documental e } \\
\text { descritiva }\end{array}$ & $\begin{array}{l}\text { Análise de } 61 \text { prontuários de crianças e adolescentes com idade } \\
\text { entre um mês e } 17 \text { anos com cateter venoso central do tipo } \\
\text { Porth o Cath. Desse total nove cateteres foram retirados por } \\
\text { conta de complicações (infecções), conclusão ou desistência do } \\
\text { tratamento. }\end{array}$ \\
\hline & $\begin{array}{l}\text { Manuseio de } \\
\text { Cateter Venoso } \\
\text { Central De Longa } \\
\text { Permanência Em } \\
\text { Pacientes } \\
\text { Portadores de } \\
\text { Câncer }\end{array}$ & $\begin{array}{l}\text { (SOUZA et al., } \\
2013 \text { ) }\end{array}$ & 013 & $\begin{array}{l}\text { Método Survey } \\
\text { (investigação } \\
\text { orientada) }\end{array}$ & $\begin{array}{l}\text { Artigo realizado com } 9 \text { enfermeiros que analisou qual é o } \\
\text { conhecimento a respeito do manuseio, medidas de prevenção e } \\
\text { tratamento das complicações relacionadas ao mesmo por } \\
\text { profissionais de enfermagem. Obstruções, infecções, } \\
\text { extravasamento, trombose e deslocamento foram algumas das } \\
\text { complicações citadas pelos entrevistados. }\end{array}$ \\
\hline \multirow[t]{2}{*}{ 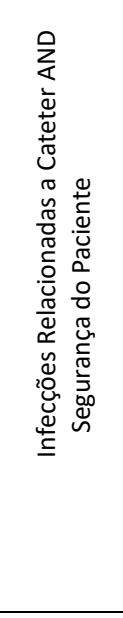 } & $\begin{array}{l}\text { Saberes da Equipe } \\
\text { de Enfermagem } \\
\text { Sobre Cuidados } \\
\text { com Cateter } \\
\text { Venoso Central }\end{array}$ & $\begin{array}{l}\text { (BARBOSA et } \\
\text { al., 2017) }\end{array}$ & 017 & $\begin{array}{l}\text { Estudo } \\
\text { Quantitativo } \\
\text { Descritivo } \\
\text { Exploratório } \\
\text { Transversal }\end{array}$ & $\begin{array}{l}\text { Participaram da seguinte pesquisa } 107 \text { profissionais de } \\
\text { enfermagem sendo que, } 81 \%(87) \text { eram técnicos de enfermagem } \\
\text { e } 19 \%(20) \text { eram enfermeiros, sendo que } 100 \% \text { dos enfermeiros } \\
\text { e } 24 \% \text { dos técnicos de enfermagem declararam realizar a prática } \\
\text { de troca do curativo do CVC. } \\
\text { Os resultados encontrados após a pesquisa estruturada por } \\
\text { questionário, foi: } \\
\text { Conhecimento: } 70 \% \text { enfermeiros e } 38 \% \text { dos técnicos obtiveram } \\
75 \% \text { (satisfatório) ou mais acertos, caracterizando } 44 \% \text { dos } \\
\text { profissionais avaliados apresentaram conhecimento suficiente } \\
\text { sobre o protocolo e } 56 \% \text {, conhecimento deficiente. }\end{array}$ \\
\hline & $\begin{array}{l}\text { Enfermagem na } \\
\text { Prevenção de } \\
\text { Infecções em } \\
\text { Cateter Totalmente } \\
\text { Implantado no } \\
\text { Paciente } \\
\text { Oncológico }\end{array}$ & $\begin{array}{l}\text { (OLIVEIRA et } \\
\text { al., } \\
\text { RODRIGUES, } \\
\text { 2016) }\end{array}$ & 016 & $\begin{array}{l}\text { Estudo de } \\
\text { Avaliação, uma } \\
\text { Revisão } \\
\text { Sistemática. }\end{array}$ & $\begin{array}{l}\text { As ações de cuidado do enfermeiro com o Cateter Venoso } \\
\text { Central Totalmente Implantado, que apesar de simples, exigem } \\
\text { da equipe a prática de medidas para evitar as possíveis } \\
\text { complicações relacionadas ao manuseio desse cateter, que } \\
\text { partem desde o princípio da lavagem das mãos até a troca a } \\
\text { manipulação do cateter. }\end{array}$ \\
\hline 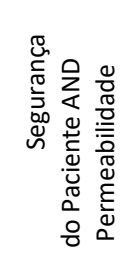 & $\begin{array}{l}\text { Manutenção da } \\
\text { Permeabilidade } \\
\text { dos Dispositivos de } \\
\text { Acesso Venoso } \\
\text { Central em } \\
\text { Pacientes com } \\
\text { Câncer. }\end{array}$ & $\begin{array}{l}\text { (CABREIRA } \\
\text { al., 2019) }\end{array}$ & 019 & $\begin{array}{l}\text { Estudo } \\
\text { Revisão } \\
\text { Sistemática }\end{array}$ & $\begin{array}{l}\text { A oclusão é uma das principais complicações quando } \\
\text { relacionada ao dispositivo de acesso vascular central, tendo } \\
\text { como um bom resultado a implementação de protocolos } \\
\text { institucionais de Flushing e Locking. }\end{array}$ \\
\hline
\end{tabular}

Tanto para Ortolani et al. (2013), Barbosa (2017), Alcântara (2019), quanto Cabreira (2019), 
observam-se a necessidade da prática de educação permanente frente ao manuseio desses cateteres, decorrentes ao seu uso em oncologia. Complicações associadas à infecção, tração acidental, obstrução, ruptura, trombose, extravasamento é descrito por Santana et al. (2018), Souza (2013). Independente do tipo de cateter escolhido, apontam os agravos na inserção de PICC, CVC e CVC-TI.

Menezes et al. (2013) relatam sobre o efeito imunossupressor da quimioterapia, o que pode favorecer o aumento do índice de infecção, além de outros fatores, como a inadequação do material do sistema de infusão fechado e manipulação excessiva do curativo sem periodicidade, abaixo de 7 dias de acordo com as guidelines e trocas de agulhas em não conformidade.

Nesse sentido, o papel do enfermeiro é baseado no apoio ao cuidado do paciente oncológico, na assistência clínica ao manuseio do cateter e suas particularidades diante dessa situação, a lei federal $\mathrm{n}^{\circ}$ 7.498/1986 regulamenta o exercício de enfermagem e compreende como competência privativa do enfermeiro os cuidados de maior complexidade técnica e que exijam conhecimentos de base científica e capacidade de tomar decisões imediatas, segundo o Conselho Federal de Enfermagem (COFEN), citado por Alcântara (2019).

De acordo com os apontamentos levantados por Barbosa (2017) há o conhecimento dos profissionais de enfermagem que tal procedimento é uma atividade exclusiva do enfermeiro, porém, devido o número reduzido de profissionais, a manipulação e troca de curativos destes acessos são efetivados por técnicos de enfermagem. Entretanto, a priorização desse procedimento é invisibilizada pelo mesmo.

Para Santana et al. (2018) a taxa de infecção em ambientes ambulatoriais é menor comparada a pacientes em tratamento quimioterápico em âmbito hospitalar, o que é evidenciado na procedência de pacientes críticos, abrangência hospitalar, fluxo ampliado de internados e leitos se contraposto ao regime ambulatorial. Dados apresentados por Cabreira (2019), retratam a importância atribuída à manutenção da permeabilidade destes cateteres, atribuição diretamente relacionada á qualidade de vida destes pacientes, uma vez que além do tratamento da quimioterapia estes dispositivos são utilizados para coleta de sangue, administração de medicamentos, como analgésicos e antibióticos

Percebe-se a necessidade da revisão de protocolos, treinamento de equipe, atualização técnicocientífica constante de acordo com os resultados expostos, a fim de propiciar um cuidado humanizado, na tentativa de minimizar ao máximo risco ao paciente. A Sistematização da Assistência de Enfermagem (SAE) vem como instrumento de qualidade no gerenciamento do cuidado, para Santana et al. (2018) e Barbosa (2017), a padronização da assistência qualifica as intervenções de enfermagem.

\section{CONCLUSÕES}

O uso destes dispositivos intravasculares está cada vez mais presente em pacientes oncológicos, principalmente o PICC (Cateter Central de Inserção Periférica), CVC (Cateter Venoso Central) e Cateter Venoso Central de Longa Permanência, frente a isso os estudos alcançados apresentam a necessidade do cuidado de enfermagem e complexidade no manuseio dos mesmos. A adoção de práticas de educação 
continuada, bem como a atualização técnico-científica dos profissionais demonstram a eficácia no tratamento dos pacientes em uso de cateteres, além de prevenir infecções, obstruções, trações e outras complicações inerentes à manipulação incorreta, com isso se tem uma redução significativa em gastos desnecessários e aumento de vida útil do cateter.

Os relatos supracitados nos estudos evidenciam o déficit de conhecimento aos diversos tipos de cateteres e condutas a serem seguidas, a implementação de protocolos nas unidades de saúde se faz necessário, a fim de proporcionar melhora na qualidade da assistência prestada, diminuindo riscos aos pacientes e possibilitando o atendimento humanizado.

No desenvolvimento da pesquisa a indisponibilidade de artigos presentes na literatura, trouxe em discussão, a necessidade de ampliação no período de busca para estudos publicados nos últimos dez anos. A escassez de publicações realizadas por enfermeiros pertencentes a dispositivos endovasculares apresentam dificuldade na elucidação de informações e consequentemente lacunas de conhecimentos e formação de protocolos eficazes. Faz-se necessário o desenvolvimento de novas pesquisas para o fornecimento de dados científicos em enfermagem, com intenção de ampliar a assistência baseada em evidência.

\section{REFERÊNCIAS}

ALCÂNTARA, D. C.. Artigo Revisão Integrativa Cateter Central De Inserção Periférica: contribuições Para a Enfermagem Oncológica. Revista de Enfermagem UFPE, v.13, n.3, p.715731, 2019. DOI: https://doi.org/10.5205/1981-8963v13i03a236058p715-731-2019

BORTOLI, P. S.. Cateter venoso central de inserção periférica em oncologia pediátrica: revisão de escopo. Acta Paulista de Enfermagem, v.32, n.2, p.220-228, 2019. DOI: https://doi.org/10.1590/1982-0194201900030

CABREIRA, V. F.. Manutenção da permeabilidade dos dispositivos de acesso venoso central em pacientes com câncer. Revista Enfermagem UERJ, v.27, 2019. DOI: https://doi.org/10.12957/reuerj.2019.39230

FONSECA, D. F.. Protocolo de cuidados com cateter venoso totalmente implantado: uma construção coletiva. Revista Texto \& Contexto Enfermagem, v.28, p.1-16, 2019. DOI http://dx.doi.org/10.1590/1980-265X-TCE-2018-0352

MENEZES, V.; BITTENCOURT, A.; MENEZES, M.. Infection Related To Central Venous Catheter: Indicator of Quality of Care in Oncology. Cuidado é Fundamental, v.5, n.3, p.373385, 2013. DOI: https://doi.org/10.9789/21755361.2013.v5i3.373-385

OLIVEIRA, T. F.; RODRIGUES, M. C. S.. Enfermagem na prevenção de infecção em cateter totalmente implantado no paciente oncológico. Cogitare enferm, v.21, n.2, p.1-5, 2016. DOI: http://dx.doi.org/10.5380/ce.v21i2.45523

ORTOLANI, L.; GASPARINO, R. C.; TRALDI, M. C. Complicações associadas ao uso de cateter totalmente implantável em crianças e adolescentes. Revista Brasileira de Cancerologia, v.59, n.1, p.51-56, 2013

SANTANA, F. G.; DIAS, P. L. M.. Cateter central de inserção periférica em oncologia pediátrica: um estudo retrospectivo. Revista Brasileira de Cancerologia, v.64, n.3, p.341-347, 2018. DOI: https://doi.org/10.32635/21769745.RBC.2018v64n3.34

SANTOS, L. M.. Fatores de risco para complicações locais da terapia intravenosa em crianças e adolescentes com câncer. Revista Brasileira de Enfermagem REBEN, v.73, n.4, p.1-9, 2020. DOI: https://doi.org/10.1590/0034-7167-2019-0471

SOUZA, G. S.. Manuseio de cateter venoso central de longa permanência em pacientes portadores de Câncer. Revista de enfermagem do Centro Oeste Mineiro, v.3, n.1, p.577-586, 2013.

BARBOSA, C. V.. Saberes da equipe de enfermagem sobre cuidados com cateter venoso central. Revol Revista de Enfermagem UFPE, v.11, n.11, 2017. DOI: http://doi.org/10.5205/reuol.23542-49901-ED.1111201710 